\title{
Cyclospora infection linked to travel to Mexico, June to September 2015
}

GL Nichols $^{1}$, J Freedman ${ }^{1}$, KG Pollock ${ }^{2}$, C Rumble ${ }^{1}$, RM Chalmers ${ }^{3}$, P Chiodini ${ }^{4}$, G Hawkins ${ }^{2}$, CL Alexander ${ }^{5}$, G Godbole $^{14}$, C Williams $^{6}$, HA Kirkbride ${ }^{1}$, M Hamel $^{7}$, Ji Hawker ${ }^{1}$

1. National Infection Service, Public Health England, London, United Kingdom

2. Health Protection Scotland, Glasgow, United Kingdom

3. Cryptosporidium Reference Unit, Public Health Wales, Swansea, United Kingdom

4. PHE National Parasitology Reference Laboratory, Hospital for Tropical Diseases, London, United Kingdom

5. Scottish Parasite Diagnostic and Reference Laboratory, Glasgow, United Kingdom

6. Public Health Wales, Cardiff, United Kingdom

7. Public Health Agency of Canada, Ottawa, Canada

Correspondence: Gordon Nichols (gordon.nichols@phe.gov.uk)

Citation style for this article:

Nichols GL, Freedman J, Pollock KG, Rumble C, Chalmers RM, Chiodini P, Hawkins G, Alexander CL, Godbole G, Williams C, Kirkbride HA, Hamel M, Hawker JI. Cyclospora infection linked to travel to Mexico, June to September 2015. Euro Surveill. 2015;20(43):pii=30048. DOI: http://dx.doi.org/10.2807/1560-7917.

ES.2015.20.43.30048

Cyclospora cayetanensis was identified in 176 returned travellers from the Riviera Maya region of Mexico between 1 June and 22 September 2015; 79 in the United Kingdom (UK) and 97 in Canada. UK cases completed a food exposure questionnaire. This increase in reported Cyclospora cases highlights risks of gastrointestinal infections through travelling, limitations in Cyclospora surveillance and the need for improved hygiene in the production of food consumed in holiday resorts.

On 14 July 2015, Health Protection Scotland (HPS) identified an unusual increase in Cyclospora infections in travellers to Mexico. National and international partners were informed and upon further investigation, a total of 176 cases have been identified in England, Scotland, Wales and Canada. An outbreak control team managed the investigation in the United Kingdom (UK). UK patients were interviewed about travel history, food consumption, clinical symptoms and demography using a questionnaire. The majority of cases had travelled to the Riviera Maya region of Mexico.

\section{Investigation of UK cases}

Cyclospora cases were identified in primary clinical diagnostic and commercial laboratories by microscopy or molecular testing. Cases were confirmed in reference laboratories using microscopic methods (e.g. examination of a wet preparation by bright field microscopy and, if structures resembling Cyclospora were observed, viewing under UV light for autofluorescence). In addition, smears were permanently stained using modified Ziehl Neelsen and examined.

In the UK, probable cases were defined as individuals with onset of gastrointestinal (GI) symptoms or a specimen date on or after 1 June 2015, travel to Mexico in the previous 14 days and $C$. cayetanensis oocysts identified in stool specimens by a local diagnostic laboratory. Confirmed UK cases were probable cases confirmed microscopically by national reference laboratories. Cases without either local or national reference laboratory confirmation were excluded from this analysis. No cases associated with travel to Mexico were identified in the UK in 2015 before 1 June.

\section{Outbreak description}

Between 1 June and 22 September 2015, 79 probable or confirmed case-patients (hereafter called cases) were reported in England $(n=55)$, Scotland $(n=21)$ and Wales $(n=3)$. No further cases linked to Mexico have been identified in the period since that date (as at 28 October 2015). Symptom onset dates were available for 62 confirmed or probable cases and ranged from 8 June to 19 August 2015 (Figure 1). Travel information was available for 60 cases; the earliest departure date from the UK to Mexico was 22 May and the latest date of return was 30 August 2015. The median age of cases was 44 years (range: 15-66) with 46 of 79 cases 40 years and older; 43 of 79 were female. Only 43 of the 79 cases diagnosed in local laboratories were confirmed by a reference laboratory.

Cases occurred over an extended period and in people who stayed at 32 different hotels on the Riviera Maya coast of Mexico, from Cancun to Tulum. A formal epidemiological study was not therefore possible. Questionnaires were completed for 46 of 79 cases, with 43 reporting all-inclusive catering, of whom 24 (56\%) reported also eating outside their hotel. 


\section{FIGURE 1}

Epidemic curve of Cyclospora cases by onset date, United Kingdom, 1 June-24 August 2015 (n = 62)

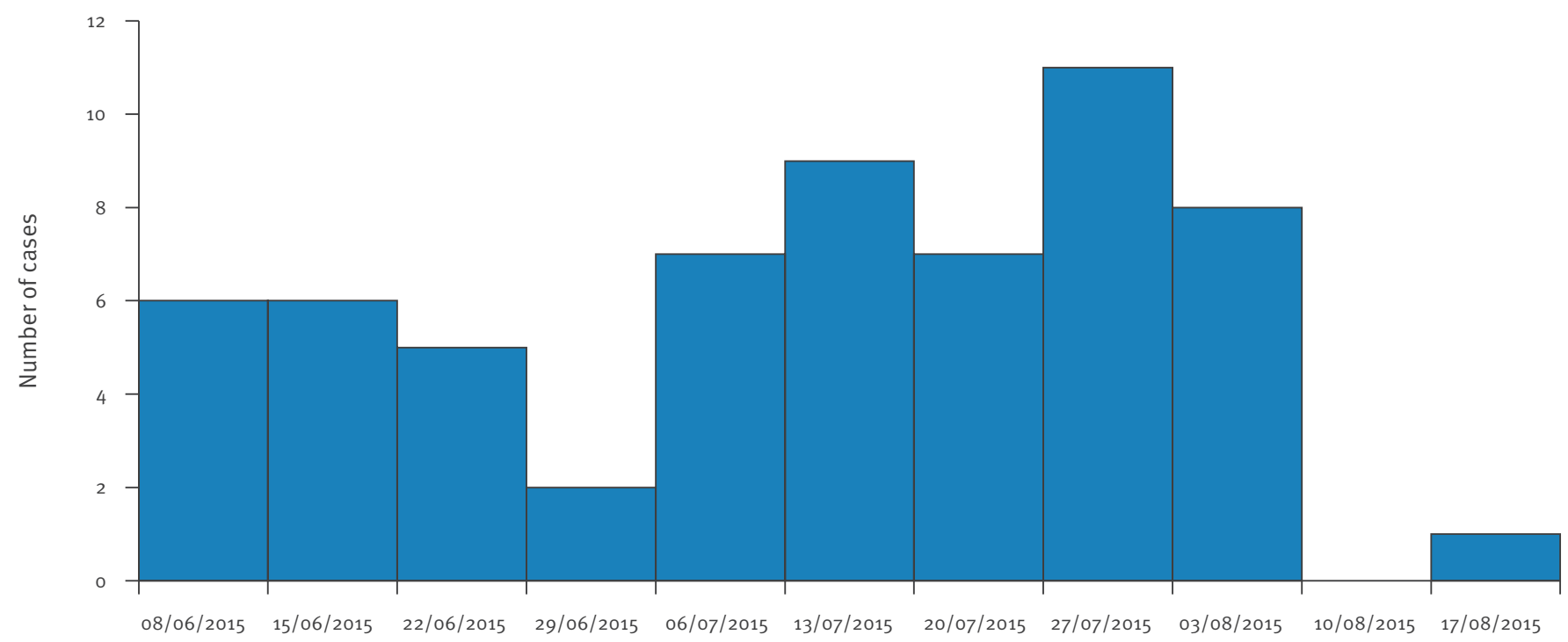

Onset date week beginning

\section{FIGURE 2}

Cyclospora cases reported per week to national surveillance, England and Wales, 1995-2014 (n = 923)

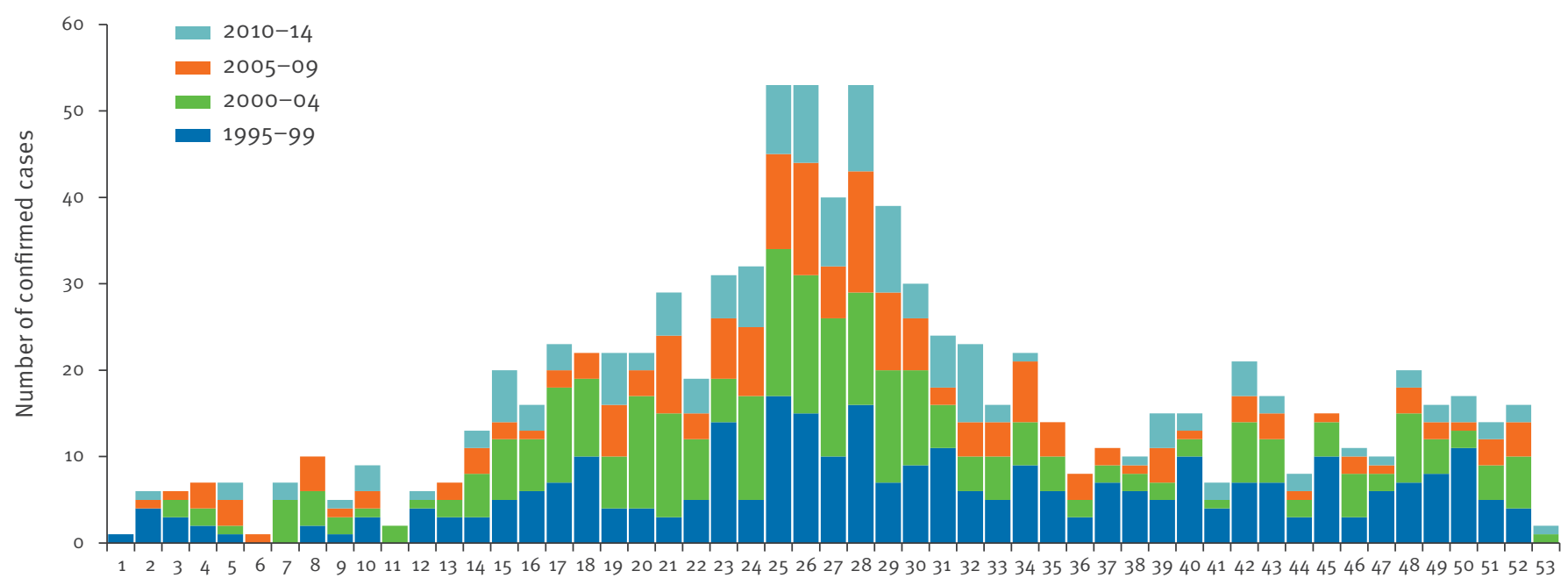

Week of specimen

Of 44 cases with symptom details recorded, all had diarrhoea (range: 5-62 days; mean: 16 days) which was rapid onset in 30. Other symptoms included abdominal pain $(n=35)$, fatigue $(n=31)$, nausea $(n=27)$, vomiting $(n=24)$, fever $(n=19)$, weight loss $(n=19)$ and headache $(n=17)$. There were no hospitalisations or deaths.

Of 45 cases for whom food histories were available, 43 consumed fruit or berries, 41 consumed salad or vegetables and 35 consumed fresh herbs. Specific items mentioned by cases included fresh mint in drinks $(n=15)$, strawberries or raspberries $(n=9)$ and coriander $(n=6)$. Most cases ate from all-inclusive buffets which also included a number of meat and fish products, cheese and desserts. Consumption of bottled water and ice was reported by 39 and 38 cases, respectively.

Awareness was raised among laboratories and public health professionals by circulating diagnostic aid sheets and travel advice and communicating with health authorities in Mexico, UK tour operators, the European Centre for Disease Prevention and Control, the Public Health Agency of Canada, the United States (US) Centers for Disease Control and Prevention (CDC) and European Union countries. 


\section{FIGURE 3}

Region visited by travel-related Cyclospora cases, England and Wales, 1995-2014 (n = 923)

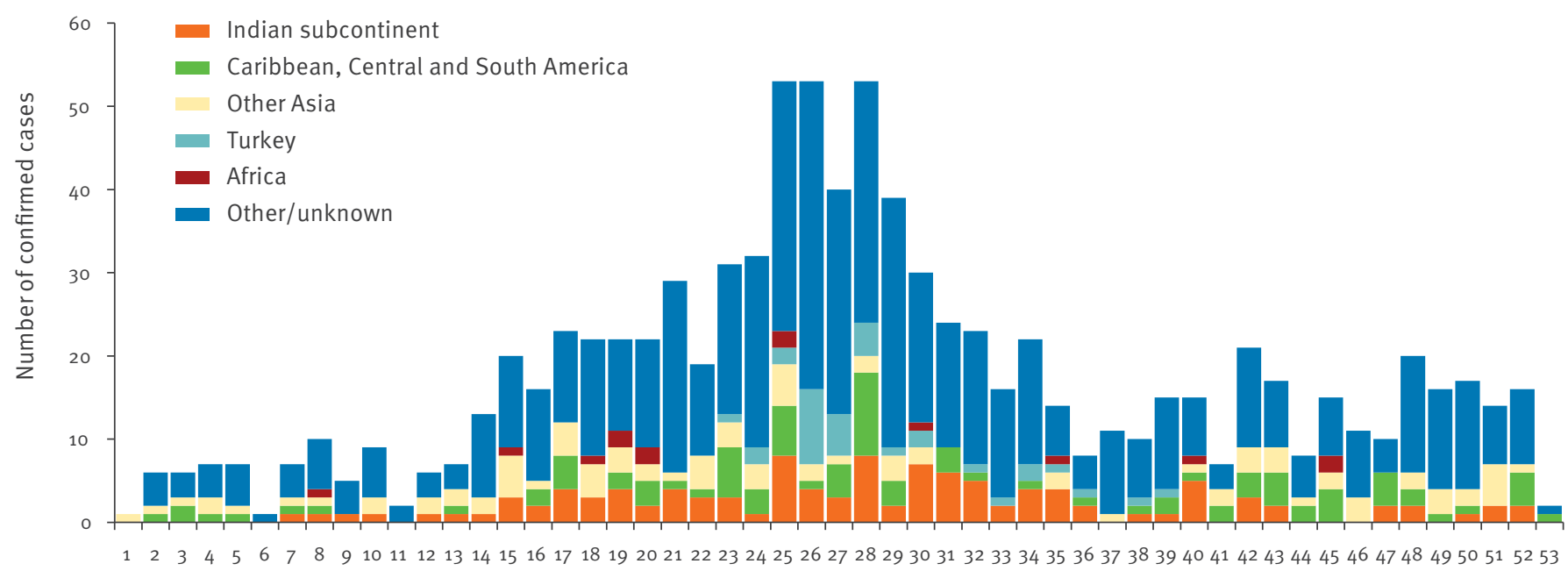

Week of year $1995-2014$

\section{FIGURE 4}

Age and sex distribution of Cyclospora cases reported to national surveillance, England and Wales, 1995-2014 $(\mathrm{n}=658)$

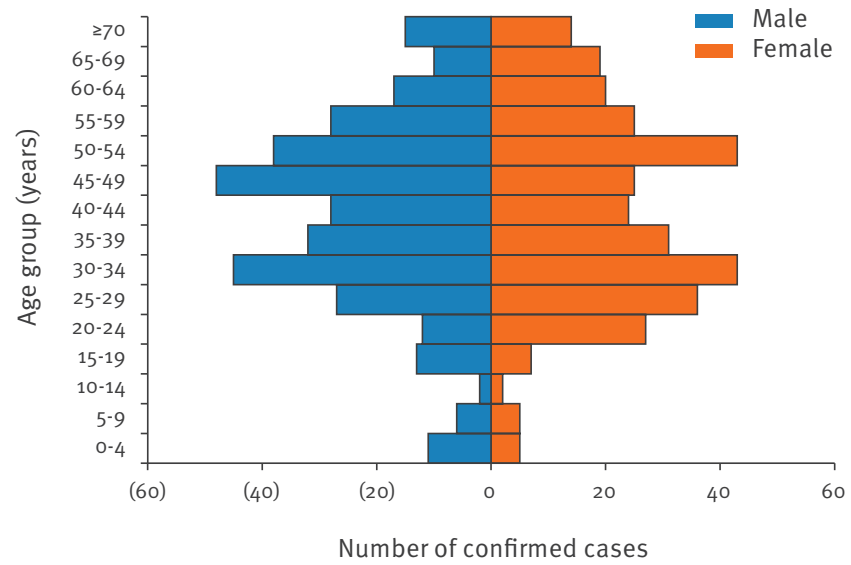

\section{Discussion}

Cyclospora cayetanensis is a protozoan parasite that causes treatable diarrhoea [1-3], and predominantly occurs in tropical and subtropical countries [4-6]. Cyclospora oocysts sporulate 10 days after being defecated and become infectious. Outbreaks of cyclosporiasis [3,7], have been linked to contaminated snow peas [8], basil [9], salad/herbs [10], raspberries and other berries $[3,11,12]$, and drinking water $[13,14]$. Sporadic infections follow travel to endemic countries, including Mexico [15-17], and imported basil from Mexico was implicated in an outbreak in Canada [18].

An increase in cyclosporiasis has been observed in UK travellers to Mexico this summer. A similar increase has also been noted in Canada: while Canada has no routine travel surveillance, 97 cases of Cyclospora infection in travellers returning from Mexico were reported from May to August 2015; the cases reporting staying at various resorts in the same geographical area as the UK cases. The UK and Canadian cases occurred in people returning from at least 36 hotels on the Riviera Maya coast of Mexico. Drinking water was an unlikely source as several different water networks supply the resorts (some hotels have their own borehole and treatment). Geographical and temporal associations suggest that the outbreak was related to a consumed product(s) distributed throughout the region rather than hygiene deficiencies in individual hotels. A multistate outbreak of cyclosporiasis has also occurred in the US, concurrent with our investigations, in which fresh cilantro from Puebla, Mexico has been implicated as the cause of cluster-associated cases in three US states [19]. Local investigation in Mexico suggests fresh cilantro from Puebla had been distributed to hotels in the Riviera Maya region. Food safety control measures have since been implemented by the Mexican authorities to ensure the safety of cilantro from Puebla state (personal communication to Public Health England: National Focal Point for Emergency INFOSAN in Mexico and National Focal Point for IHR, Mexico, 6 October 2015).

Cyclospora infections are seasonal in England and Wales (Figure 2). Where travel history is known, travel to the Indian subcontinent, Turkey, the Caribbean, Central and South America is commonly reported (Figure 3). Childhood infections are uncommon and case numbers in male and female patients are equivalent (Figure 4). Eleven laboratories detected five or more cases between 2005 and 2014, and many laboratories had no detections. Cyclospora oocysts can be detected readily by microscopy, but if screening algorithms are not 
followed, cases can go undetected. National External Quality Assessment for Cyclospora has improved from $23 \%$ in the mid-1990s to $86 \%$ by 2011 (personal communication: UK National External Quality Assessment Service, 18 September 2015). Limited information is available on cases in other EU Member States. The FilmArray GI Panel [20] or equivalent PCR array would facilitate faecal screening for Cyclospora infections, in part because it does not require the physician or laboratory to specifically request Cyclospora testing. Improvements are needed in hygiene control during the production and harvesting of salad and soft fruit products in countries with higher incidence [21].

\section{Acknowledgements}

This work was funded in part by the UK Medical Research Council (MRC) and UK Natural Environment Research Council (NERC) for the MEDMI Project; the National Institute for Health Research Health Protection Research Unit (NIHR HPRU) in Environmental Change and Health at the London School of Hygiene and Tropical Medicine in partnership with Public Health England (PHE), and in collaboration with the University of Exeter, University College London, and the Met Office; the NIHR HPRU in Gastrointestinal Infections; and the European Regional Development Fund Programme and European Social Fund Convergence Programme for Cornwall and the Isles of Scilly (University of Exeter Medical School), Canadian provincial and local public health authorities. PLC is supported by the National Institute for Health Research University College London Hospitals Biomedical Research Centre. The authors are grateful to staff at the national reference units for expertise in confirming diagnoses and local health protection teams for contributing to collection of risk data.

\section{Conflict of interest}

None declared.

\section{Authors' contributions}

All authors were involved in the outbreak investigation and contributed to the manuscript. This included expertise in parasitology ( $P C, G N, R C, K P, G G)$, epidemiology ( $G N, M H$, $\mathrm{RC}, J \mathrm{~J}, \mathrm{KP}, \mathrm{CR}, \mathrm{GH}, \mathrm{JH}, \mathrm{CW}$ ) and diagnosis (PC, GN, GG, CA).

\section{References}

1. ChiodiniPL. A 'new' parasite: human infection with Cyclospora cayetanensis.Trans R Soc Trop Med Hyg. 1994;88(4):369-71. DOI: 10.1016/0035-9203(94)90385-9 PMID: 7570808

2. OrtegaYR, SterlingCR, GilmanRH, CamaVA, DíazF. Cyclospora species--a new protozoan pathogen of humans.N Engl J Med. 1993;328(18):1308-12. DOI: 10.1056/NEJM199305063281804 PMID: 8469253

3. HerwaldtBL. Cyclospora cayetanensis: a review, focusing on the outbreaks of cyclosporiasis in the 1990s.Clin Infect Dis. 2000;31(4):1040-57. DOI: 10.1086/314051 PMID: 11049789

4. LeguaP, SeasC. Cystoisospora and cyclospora.Curr Opin Infect Dis. 2013;26(5):479-83.PMID: 23982239

5. OrtegaYR, SanchezR. Update on Cyclospora cayetanensis, a food-borne and waterborne parasite.Clin Microbiol Rev. 2010;23(1):218-34. DOI: 10.1128/CMR.00026-09 PMID: 20065331

6. Chacín-BonillaL. Epidemiology of Cyclospora cayetanensis: A review focusing in endemic areas.Acta Trop. 2010;115(3):18193. DOI: 10.1016/j.actatropica.2010.04.001 PMID: 20382099
7. ChalmersRM, NicholsG, RooneyR. Foodborne outbreaks of cyclosporiasis have arisen in North America. Is the United Kingdom at risk?Commun Dis Public Health. 2000;3(1):50-5.

8. Centers for Disease Control and Prevention (CDC),. Outbreak of cyclosporiasis associated with snow peas--Pennsylvania, 2004.MMWR Morb Mortal Wkly Rep. 2004;53(37):876-8.PMID: 15385921

9. HoangLM, FyfeM, OngC, HarbJ, ChampagneS, DixonB, et al. Outbreak of cyclosporiasis in British Columbia associated with imported Thai basil. Epidemiol Infect. 2005;133(1):23-7. DOI: 10.1017/So950268804003176 PMID: 15724706

10. DöllerPC, DietrichK, FilippN, BrockmannS, DreweckC, VontheinR, et al. Cyclosporiasis outbreak in Germany associated with the consumption of salad. Emerg Infect Dis. 2002;8(9):992-4. DOI: 10.3201/eido809.010517 PMID: 12194782

11. HoAY, LopezAS, EberhartMG, LevensonR, FinkelBS, da SilvaAJ, et al. Outbreak of cyclosporiasis associated with imported raspberries, Philadelphia, Pennsylvania, 2000. Emerg Infect Dis. 2002;8(8):783-8. DOI: 10.3201/eido808.020012 PMID: 12141962

12. ManuelD, NeamatullahS, ShahinR, ReymondD, KeystoneJ, CarlsonJ, et al. An outbreak of cyclosporiasis in 1996 associated with consumption of fresh berries- Ontario. Can J Infect Dis. 2000;11(2):86-92.PMID: 18159270

13. AksoyU, AkisuC, SahinS, UslucaS, YalcinG, KuralayF, et al. First reported waterborne outbreak of cryptosporidiosis with Cyclospora co-infection in Turkey. Euro Surveill. 2007;12(2):3142.

14. BaldurssonS, KaranisP. Waterborne transmission of protozoan parasites: review of worldwide outbreaks - an update 2004-2010.Water Res. 2011;45(20):6603-14. DOI: 10.1016/j. watres.2011.10.013 PMID: 22048017

15. DiazE, MondragonJ, RamirezE, BernalR. Epidemiology and control of intestinal parasites with nitazoxanide in children in Mexico.Am J Trop Med Hyg. 2003;68(4):384-5.PMID: 12875284

16. Sánchez-VegaJT, Cabrera-FuentesHA, Romero-OlmedoAJ, OrtizFríasJL, SokolinaF, BarretoG. Cyclospora cayetanensis: this emerging protozoan pathogen in Mexico.Am J Trop Med Hyg. 2014;90(2):351-3. DOI: 10.4269/ajtmh.12-0782 PMID: 24379243

17. Orozco-MosquedaGE, Martínez-LoyaOA, OrtegaYR. Cyclospora cayetanensis in a pediatric hospital in Morelia, México.Am J Trop Med Hyg. 2014;91(3):537-40. DOI: 10.4269/ajtmh.13-0535 PMID: 24957545

18. ShahL, MacDougallL, EllisA, OngC, ShyngS, LeBlancL, et al. Challenges of investigating community outbreaks of cyclosporiasis, British Columbia, Canada. Emerg Infect Dis. 2009;15(8):1286-8. DOI: 10.3201/eid1508.081585 PMID: 19751593

19. Centers for Disease Control and Prevention (CDC). Cyclosporiasis Outbreak Investigations - United States, 2015. Atlanta: CDC. [Accessed:22 Oct 2015]. Available from: http:// www.cdc.gov/parasites/cyclosporiasis/outbreaks/2015/index. html

20. FilmArray Gastrointestinall Panel Product Sheet. Salt Lake City: BioFire Diagnostics. [Accessed: 22 Oct 2015]. Available from: http://filmarray.com/assets/pdf/Info-Sheet-GI-PanelMRKT-PRT-0234-07.pdf

21. McClure P, Ronnie N, Waskar M. HACCP, risk assessment, and risk communication. In: Robertson LJ, Smith HV, eds. Foodborne Protozoan Parasites. Hauppauge: Nova Science; 2012. pp. 239-66. 